Canad. Math. Bull. Vol. 20 (1), 1977

\title{
APPROXIMATION BY GENERALISED POLYNOMIALS WITH INTEGRAL COEFFICIENTS
}

\author{
BY \\ J. TZIMBALARIO
}

Let $C[0,1]$ be the space of all continuous real valued functions defined in $[0,1]$ with the supremum norm

$$
\|f\|_{\infty} \equiv \sup _{x \in[0,1]}|f(x)|
$$

The subspace of $C[0,1]$ consisting of all functions $f(x)$ for which $f(0)$ and $f(1)$ are integers will be denoted by $C_{0}[0,1]$. Let $\Lambda=\left\{\lambda_{i}\right\}_{0}^{\infty}$ be a sequence of real numbers satisfying:

$$
\lambda_{0}=0, \lambda_{i} \geq 0 \quad(i \geq 1)
$$

A $\Lambda$-polynomial is a function of the form $\sum_{i=0}^{n} x^{\lambda_{i}}$ where $a_{i}$ are real numbers. It is well known $[4,5]$ that the $\Lambda$-polynomials are dense in $C[0,1]$ provided

$$
\sum_{\lambda_{i} \neq 0} \lambda_{i}^{-1}=\infty
$$

There are several results about approximation by algebraic polynomials with integral coefficients. In $C[0,1]$ the approximation of $f(x)$ is possible if and only if $f(x) \in C_{0}[0,1]([1],[2],[3])$.

Ferguson [1], and Ferguson and von Golitschek [2] were interested to know if this result is true also for $\Lambda$-polynomials in $C_{0}[0,1]$, and they proved that the result is true under some restrictions on $\Lambda$. Here we remove the unnecessary restrictions.

THEOREM. Let $\Lambda=\left\{\lambda_{i}\right\}_{0}^{\infty}$ be a sequence of real numbers satisfying (2). The integral $\Lambda$-polynomials are dense in $C_{0}[0,1]$ if and only if

$$
\sum_{\lambda_{i} \neq 0} \lambda_{i}^{-1}=\infty
$$

In order to prove the theorem we need two lemmas.

Received by the editors October 21, 1974 and, in revised form, August 4, 1976. 
LEMMA 1. Let $\Lambda=\left\{\lambda_{i}\right\}_{0}^{\infty}$ be a sequence of real numbers satisfying

(i) $0=\lambda_{0}<\lambda_{1}<\cdots<\lambda_{n} \uparrow \infty$

(ii) $\lambda_{n} \geq n$

(iii) $\sum_{i=1}^{\infty} \lambda_{i}^{-1}=\infty$,

then the integral $\Lambda$-polynomials are dense in $C_{0}[0,1]$.

Proof. This lemma was proved in [2] under the restriction of integral $\lambda_{n}$. The proof is identical as long as $\lambda_{n} \geq n$.

LEMMA 2. Let $\lambda=\left\{\lambda_{n}\right\}$ be a strictly increasing sequence satisfying $\lambda_{n} \rightarrow \infty$ and $\sum_{\lambda_{n}>0} \lambda_{n}^{-1}=\infty$. Then, there is a subsequence $\left\{\lambda_{n_{i}}\right\}$ which satisfies

$$
\lambda_{n_{i}} \geq i
$$

and still

$$
\sum_{i=1}^{\infty} \lambda_{n_{i}}^{-1}=\infty .
$$

Proof. The construction of $\left\{\lambda_{n_{i}}\right\}$ is made by constructing two sequences of indices. Choose $m_{1}$ to be the first index for which $\lambda_{m_{1}}>0$ and $p_{1}$ to be the smallest index which satisfies

$$
p_{1}>m_{1}, \lambda_{p_{1}}<p_{1}-m_{1}
$$

Suppose that we had already chosen $m_{k}<p_{k}$. Define $m_{k+1}$ and $p_{k+1}$ to be the smallest indices such that

$$
\begin{cases}m_{k+1}>p_{k}, & \lambda_{m_{k+1}} \geq \sum_{j=1}^{k}\left(p_{j}-m_{j}\right) \\ p_{k+1}>m_{k+1}, & \lambda_{p_{k+1}}<\sum_{j=1}^{k+1}\left(p_{j}-m_{j}\right) .\end{cases}
$$

If there is no such $p_{k+1}$, stop the construction and choose $\lambda_{n_{i}}=\lambda_{m_{k+1}+i}, i \geq 1$. Here (6) and (7) are obviously satisfied. Hence we can assume that for every $k \geq 1$ there are finite $m_{k+1}$ and $p_{k+1}$ as stated in (8). In this case the sequence $\left\{\lambda_{n_{i}}\right\}$ satisfying (5) and (6) will be given by:

$$
\begin{cases}\lambda_{m_{1}+i} & \text { if } \quad 1 \leq i<p_{1}-m_{1} \\ \lambda_{m_{k+1}+i}-\sum_{j=1}^{k}\left(p_{j}-m_{j}\right) & \text { if } \quad \sum_{j=1}^{k}\left(p_{j}-m_{j}\right) \leq i<\sum_{j=1}^{k+1}\left(p_{j}-m_{j}\right) .\end{cases}
$$


It is clear that $\lambda_{n_{i}} \geq i(i \geq 1)$. Let us check if (6) is satisfied

$$
\begin{aligned}
\sum_{i=1}^{\infty} \lambda_{n_{i}}^{-1} & \geq \sum_{k=2}^{\infty} \sum_{j=m_{k}}^{p_{k}-1} \lambda_{j}^{-1} \\
& \geq \sum_{k=2}^{\infty} \frac{p_{k}-m_{k}}{\sum_{j=1}^{k}\left(p_{j}-m_{j}\right)} .
\end{aligned}
$$

The quantity $q_{k}$ defined by

$$
q_{k} \equiv \sum_{j=1}^{k}\left(p_{i}-m_{i}\right)
$$

tends to $\infty$ as $k \rightarrow \infty$. Then

$$
\sum_{j=1}^{\infty} \lambda_{n_{i}}^{-1} \geq \sum_{k=2}^{\infty} \frac{q_{k}-q_{k-1}}{q_{k}}=\sum_{k=2}^{\infty}\left(1-\frac{q_{k-1}}{q_{k}}\right) .
$$

But $\sum_{k=2}^{\infty}\left(1-\left(q_{k-1} / q_{k}\right)\right)$ diverges if and only if the product $\prod_{k-2}^{\infty}\left(q_{k} / q_{k-1}\right)$ diverges:

$$
\prod_{k=2}^{l}\left(\frac{q_{k}}{q_{k-1}}\right)=\frac{q_{l}}{q_{1}} \rightarrow \infty, \quad \text { as } \quad l \rightarrow \infty .
$$

Proof of the Theorem. The necessity of the condition (4) follows from the well known Muntz result about $\Lambda$-polynomials.

Sufficiency. If the sequence $\left\{\lambda_{l}\right\}$ has a finite limit point, the problem was solved in [2].

If the sequence $\left\{\lambda_{i}\right\}$ has no finite limit points, we can arrange it in an increasing order, and then, by Lemma 2 , there exists a subsequence $\left\{\lambda_{n_{i}}\right\}$ which satisfies $\lambda_{n_{i}} \uparrow, \lambda_{n_{i}} \geq i(i \geq 1)$, and $\sum \lambda_{n_{i}}^{-1}=\infty$. By Lemma 1 , the integral polynomials in 1 and $x^{\lambda_{n_{i}}}$ will be a dense set in $C_{0}[0,1]$.

\section{REFERENCES}

1. L. B. O. Ferguson, Muntz-Szasz theorem with integral coefficients I, Proc. Int. Conf. Madras, 1973 (to appear).

2. L. B. O. Ferguson, and M. v. Golitschek, Muntz-Szasz theorem with integral coefficients II. (to appear).

3. S. Kakeya, On approximate polynomials, Tohoku Mat. J. 6 (1914), 182-186.

4. Ch. H. Muntz, Uber den Approximationssatz von Weierstrass, Math. Abhandlungen H. A. Schwartz zu seinem 50. Doctorjubilaum gewidmet, Berlin 1914, p. 303-312.

5. R. E. A. C. Raley and N. Wiener, Fourier Transforms in the Complex Domain, A. M. S., Providence, Rhode Island 1934.

P.O. Box 341,

RISHON LE ZION 